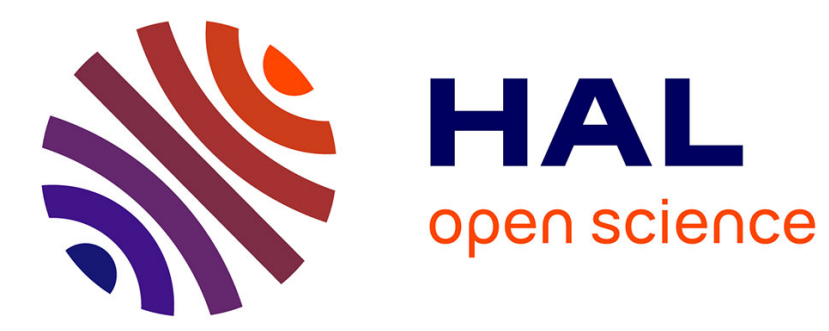

\title{
Le dernier interglaciaire et le cycle climatique würmien dans le Sud-Ouest et le Massif Central français.
}

Henri Laville, Jean-Paul Raynal, Pierre-Jean Texier

\section{To cite this version:}

Henri Laville, Jean-Paul Raynal, Pierre-Jean Texier. Le dernier interglaciaire et le cycle climatique würmien dans le Sud-Ouest et le Massif Central français.. Bulletin de l'Association française pour l'étude du Quaternaire, 1986, 1-2, pp.35-46. halshs-00005529

\section{HAL Id: halshs-00005529 \\ https://shs.hal.science/halshs-00005529}

Submitted on 13 Nov 2005

HAL is a multi-disciplinary open access archive for the deposit and dissemination of scientific research documents, whether they are published or not. The documents may come from teaching and research institutions in France or abroad, or from public or private research centers.
L'archive ouverte pluridisciplinaire $\mathbf{H A L}$, est destinée au dépôt et à la diffusion de documents scientifiques de niveau recherche, publiés ou non, émanant des établissements d'enseignement et de recherche français ou étrangers, des laboratoires publics ou privés. 


\title{
LE DERNIER INTERGLACIAIRE ET LE CYCLE CLIMATIQUE WURMIEN DANS LE SUD-OUEST ET LE MASSIF CENTRAL FRANÇAIS
}

\author{
par Henri LAVILLE *, Jean-Paul RAYNAL * et Jean-Pierre TEXIER *
}

\begin{abstract}
RESUME
Les nouveaux documents paléoclimatiques et chronologiques obtenus dans le Sud-Ouest et le Massif central français viennent préciser la chronostratigraphie des 125 derniers millénaires précédemment établie. Ils confortent également les corrélations proposées avec le domaine marin et avec les grandes séquences palynologiques continentales (Grande Pile. Les Echets). lls permettent de définir les différentes étapes de l'enchainement des phénomènes climatiques (transitions, signaux, seuils).
\end{abstract}

Mots clés : Paléoclimats. Transitions, Signaux, Seuils, Pléistocène supérieur, Dernier interglaciaire, Sud-Ouest, Massif Central, France.

\section{ABSTRACT}

\section{LAST INTERGLACIAL AND WURMIAN CLIMATIC CYCLE IN SOUTH-WEST AND MASSIF CENTRAL OF FRANCE.}

South-West and Massif Central of France provide new palaeoclimatic and chronological datas which detail the chronostratigraphy of the last 125000 years previously established. Correlations with deep-sea cores scale and long palynological continental sequences (Grande Pile, Les Echets) are thus strengthened. Different stages in the chain of climatic phaenomena are defined (transitions, signals, thresholds).

Key-words : Palaeoclimates, Transitions, Signals, Thresholds, Upper Pleistocene, Late interglacial, Late glacial, South-West, Massif Central, France.

Depuis 1956, la démarche pluridisciplinaire développée dans le Sud-Ouest de la France sous l'impulsion de F. Bordes, par l'analyse de séquences nombreuses et particulièrement détaillées, a permis de jeter les bases d'un système chronoclimatique cohérent des 150 derniers millénaires que les données obtenues dans le domaine marin voisin sont venues récemment conforter et enrichir (Laville et al., 1983).

L'adoption d'un vocabulaire précis était indispensable pour hiérarchiser les événements climatiques et clarifier le débat sur les différents systèmes chronostratigraphiques proposés et leurs corrélations réciproques; nous avons ainsi défini les termes phase, stade et interstade, glaciaire et interglaciaire (Laville et al., Laville, Raynal et Texier, 1984) (1).
L'enchaînement des faits majeurs observés et leur interprétation dynamique se trouvent aujourd'hui confortés au fur et à mesure que se découvrent d'autres séquences continentales dans des domaines géographiques voisins. Nous présentons ici une première synthèse concernant le Bassin aquitain et sa marge orientale de moyenne montagne. Les termes d'interglaciaire Riss-Würm, de Würm ancien, d'interstade würmien et de Würm récent (en partie), désignent la période étudiée.

(1) Nous écrirons interstade entre guillemets lorsqu'il est fait référence à la terminologie de la zonation pollinique pour éviter toute confusion avec le système chronologique que nous utilisons. La position chronologique du "sol de Kesselt " ayant été récemment remise en question (Haesaerts, 1984), nous utiliserons le vocable Kesselt entre guillemets pour désigner la phase d'amélioration climatique repérée entre celles d'Arcy et de Tursac.

- U.A. 133 CNRS, Institut du Quaternaire, Université de Bordeaux I, Avenue des Facultés, 33405 Talence Cedex (France). 
Tabl. 1. - Propositions de corrélations entre la séquence chronoclimatique du Sud-Ouest et du Massif Central et les séquences polliniques de la Grande Pile et des Echets, la zonation pollinique classique et les stades isotopiques océaniques.

Tabl. 1. - Attempt of correlation of the chronoclimatic succession in South-West and Massif central with the Grande Pile and les Echets pollinic sequences, the standard pollinic zonation and the oceanic isotopic stages.

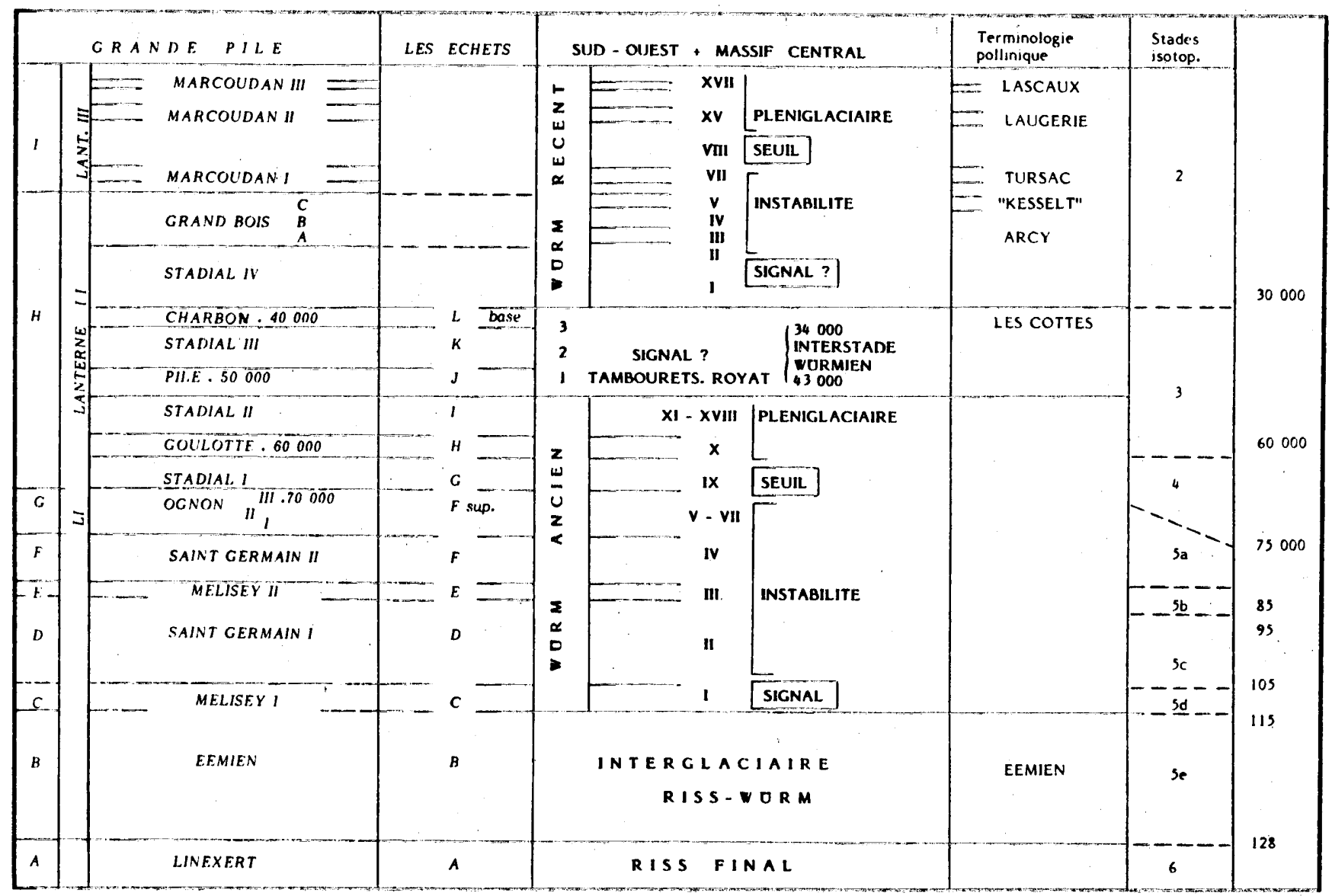

\section{LE DERNIER INTERGLACIAIRE (RISS- WURM)}

\subsection{Les données du Sud-Ouest et du Massif-Central}

Cet épisode biostasique intervient après une période exceptionnellement rigoureuse, le Riss récent (Laville et al., 1983; Texier et al., 1983). Il correspond à un événement climatique et morphologique majeur. Un surcreusement fluviatile généralisé et de grande ampleur se manifeste dans les vallées. Les différents types de dépôts (fluviatileś, de versants, de plateaux, d'entrée de grotte et d'abrissous-roche) datés du Riss récent sont affectés par une importante pédogenèse qui occasionne le développement d'épais sols lessivés bruns ou brun-rouge. Les études micromorphologiques en cours portant sur les dépôts fluviatiles de la Vézère (travaux $N$. Mémoire) et sur les dépôts colluviaux du bassin de
l'Isle et de l'Allier (travaux P. Bertran) montrent que cet épisode est responsable de la formation d'argilanes brunes ou rouges finement litées et biréfringentes. Ces dernières sont révélatrices d'un climat à précipitations régulières et d'un couvert végétal continu (Fedoroff et Tursina, 1984).

Les données polliniques précisent les caractéristiques de l'optimum climatique du Riss-Würm. Ainsi, dans la grotte Bourgeois-Delaunay (La Chaise, Charente), une formation stalagmitique polycyclique (Debenath, 1974; Debenath, Raynal, Schwarcz, 1980) a fait l'objet de douze datations UraniumThorium dont la moyenne est $151000 \pm 15000$ (Blackwell, Schwarcz, Debenath, 1983). En fait, les dates obtenues se partagent en deux groupes, l'un vers 150000 B.P., l'autre vers 120000 B.P., et suggèrent deux épisodes de construction correspondant respectivement à une phase d'amélioration du Riss III et à l'interglaciaire Riss-Würm; la lacune de sédimentation correspondrait au Riss final. La partie 
supérieure du plancher a révélé une flore de type Chênaie mixte à nombreux éléments thermophiles et à éléments méditerranéens variés (Buis, Chêne vert, Vigne, Erica mediterranea, Rhododendron ponticum ). C'est un stade d'extension de forêts denses $(\mathrm{AP}=83$ à $90 \%)$ (Paquereau, 1975).

D'autres sites permettent de suivre avec précision la dégradation climatique de la fin du Riss-Würm qui aboutit à un épisode de froid très accentué, pour nous la première manifestation du stade glaciaire Würm ancien (Laville, Raynal, Texier, 1984). Au Breuil (Dordogne) (Paquereau et Texier, 1973), on observe tout d'abord le déclin de la Chênaie, un éclaircissement des forêts et la régression concomitante du Noisetier $(A P=86 \%)$. Puis apparaissent des conditions fraîches et très humides qui favorisent l'extension du Charme et la raréfaction des éléments méditerranéens (AP $=82$ à $69 \%$ ). Peu à peu, le taux de boisement diminue et les conifères deviennent prépondérants $(A P=63 \%$ ). Enfin, les Herbacées héliophiles se multiplient et on aboutit à un stade de forêt claire où domine le Pin sylvestre accompagné de quelques Sapins, Epiceas et de rares feuillus $(\mathrm{AP}=35$ à $12 \%)$. Cette dernière phase est vraisemblablement responsable du début de l'érosion des sols Riss-Würm que l'on observe systématiquement dans toute les séquences stratigraphiques.

Une évolution identique a été reconnue dans le remplissage de la dépression de maar des Rivaux (Haute-Loire, alt. $640 \mathrm{~m}$ N.G.F.) (Raynal et al., 1981). Les dépôts du terme $E$ de la séquence (formation de versant) se mettent en place sous climat froid et sec, stade très ouvert de prairies rases et de steppes (AP $=8$ à $9 \%$; Pin sylvestre dominant), rapporté au Riss final ("Riss III "). Ils sont recouverts par les formations lacustres du terme $E$ dont l'étude palynologique caractérise la fin d'une période interglaciaire : flore arbustive très variée à la base $(A P=63 \%)$, riche en conifères (Sapin 12\%, Epicea $10 \%$, Pin sylvestre $7 \%$ ) et les feuillus (Bouleau, Aulne, Noisetier, Chêne, Saule, Orme, Tilleul : $34 \%)$. On note ensuite une disparition progressive des feuillus thermophiles, une progression du Pin sylvestre (12 à $23 \%$ ), la régression du Sapin (21 à $4 \%$ ) et de l'Epicea (7 à $2 \%$ ); AP passe de 59 à $40 \%$. Ces dépôts sont alors riches en revêtements pédologiques remaniés ("papules ") qui témoignent de l'amorce de la déstabilisation des versants et du début de l'érosion des sols interglaciaires.

\subsection{Corrélations}

L'interglaciaire Riss-Würm ainsi défini, se corrèle avec le sous-stade 5e (128000-115000 ans B.P.) de la courbe isotopique de l'oxygène établie en milieu océanique et avec l'Eémien du système chronologique de l'Europe du Nord. Cet épisode climatique a été retrouvé par J.-L. Turon (1984) au large des côtes d'Aquitaine sur la base de l'étude sporo-pollinique de carottes marines. Nous soulignerons cependant que cet auteur étend l'interglaciaire à l'ensemble du stade 5 bien que, comme nous en domaine continental, il observe après le sous-stade $5 e$, une oscillation froide brutale et importante qu'il assimile au sous-stade $5 \mathrm{~d}$ de la courbe de l'oxygène.

D'autre part, les caractéristiques paléobotaniques de l'interglaciaire Riss-Würm mises en évidence en Aquitaine (cf. supra) autorisent une corrélation avec la zone pollinique B de la Grande Pile (Woillard, 1978, 1980) ainsi qu'avec la zone B du site des Echets (De Beaulieu et Reille, 1984).

\section{LE STADE GLACIAIRE WÜRM ANCIEN}

\subsection{Les données du Sud-Ouest et du Massif Central}

A l'intérieur du Würm ancien, 18 phases climatiques distinctes ont été identifiées sur la base de données sédimentologiques (Laville, 1975), paléontologiques et paléobotaniques (Bordes et Prat, 1965; Bordes, Laville et Paquereau, 1969; Paquereau, 1974-75).

La phase I est une oscillation brutale à caractère froid très accentué, interprétée comme une crise climatique, signal du début de la glaciation würmienne. En effet, bien qu'elle soit suivie d'améliorations climatiques notables (Phases II et IV en particulier), on ne retrouve plus ensuite de conditions aussi favorables que celles de l'interglaciaire Riss-Würm. De plus, elle marque une rupture des équilibres physiques et biologiques interglaciaires et provoque ainsi le déclenchement de phénomènes sédimentaires de type périglaciaire : solifluxions et cryoturbations dans les dépôts de recouvrement, gélivation très active dans les entrées de grottes et les abris-sous-roche. La partie supérieure des sols interglaciaires est profondément érodée. La palynologie confirme cette modification radicale des paléoenvironnements et indique un paysage végétal très déboisé $(\mathrm{AP}=10 \%)$ à Pin sylvestre dominant et à nombreuses Herbacées caractéristiques des pelouses sèches. Cet événement se traduit dans la faune par l'apparition du Renne accompagné du Chamois.

Ensuite, jusqu'à la phase VIII incluse, alternent dans le Sud-Ouest des périodes d'adoucissement et des périodes froides. Cependant, les améliorations 
des phases II, IV, VI et VIII sont de moins en moins marquées thermiquement, alors que les phases III, $V$ et VII qui les séparent sont de plus en plus froides et de plus en plus sèches. Dans les dépôts de plein air cette période se traduit principalement par des phénomènes colluviaux; dans les entrées de grottes, on observe la superposition de dépôts ruisselés et de dépôts cryoclastiques. La faune est alors dominée par des espèces sylvicoles (Chevreuil, Sanglier); elle comprend également un Cerf de petite taille qui contraste de façon remarquable avec celui de grande taille contemporain du Riss supérieur (Prat et Suire, 1971). Le Renne n'apparait que timidement et seulement lors des phases les plus froides. Les données de la palynologie confortent ce schéma évolutif du climat : caractère tempéré assez accentué des phases II et IV $(\mathrm{AP}=60 \%)$, s'atténuant nettement lors des deux améliorations suivantes des phases VI et VIII (AP $\leqslant 33 \%$ ); froid modéré lors de la phase III $(\mathrm{AP}=13$ à $16 \%)$ devenant progressivement plus vif lors des phases $\mathrm{V}$ et $\mathrm{VII}(\mathrm{AP}=10 \%)$.

La phase IX est très rigoureuse $(\mathrm{AP}=10$ à $8 \%)$. Elle constitue un seuil climatique. Elle provoque en effet un changement profond et durable des paléoenvironnements. On assiste à la réapparition d'un Cerf de grande taille et, à partir de cet épisode, le Renne et le Bouquetin vont jouer un rôle important dans les assemblages fauniques. Un nouveau type de flore apparait dans lequel dominent Ephedra, Galium, Armeria et Poterium. Ces genres s'ajoutent aux Hélianthèmes et aux Armoises déjà présentes. Les paysages végétaux sont très ouverts et les espèces héliophiles et steppiques largement prépondérantes. Cette détérioration climatique se marque également dans la sédimentation par une accentuation des phénomènes de type périglaciaire qui prévaudront largement jusqu'à l'interstade würmien.

Une évolution comparable est enregistrée en Basse-Auvergne et Velay. Un exemple type est celui du remplissage lacustre de la dépression du maar de Saint-Hippolyte (Puy-de-Dôme, altitude $460 \mathrm{~m}$ N.G.F.) (Raynal et al., 1984, 1985). L'explosion phréatomagmatique responsable du creusement de la cuvette intervient pendant la transition de la phase II à la phase III du Würm ancien (Cler TL 50 : $94000 \pm 14000$ avant 1980). Une puissante banquette lacustre littorale commence ensuite à s'édifier aux dépens du croissant de pyroclastites. Après la dernière phase tempérée du Würm ancien $(\mathrm{AP}=39$ à $33 \%$, Conifères dominants, quelques feuillus), on observe l'instauration progressive de conditions pléniglaciaires. On note alors dans la flore l'apparition d'espèces steppiques (Hélianthemum, Galium, Arméria). La détérioration climatique s'accentue vers des conditions très rigoureuses et sèches (apparition d'Ephedra) qui s'affirment avec un stade presque complètement déboisé évoquant une phase glaciaire majeure à son maximum. Ces caractères, compte tenu de la différence de situation géographique des sites, se corrèlent parfaitement avec ceux de la phase IX du Würm ancien du Sud-Ouest et constituent encore ici un seuil climatique.

Le sommet des dépôts montre un léger retour à des conditions plus humides et moins sévères qui pourraient représenter l'amorce de la phase $X$ du Würm ancien.

Au cours de la première partie du Würm ancien dans le Sud-Ouest de la France, les assemblages fauniques sont dominés par le Cerf suivi du Chevreuil et du Sanglier. Puis, sous des conditions sensiblement plus rigoureuses, apparaît timidement le Renne et se développent le Cheval et les Bovinés. A Saint-Hippolyte, la faune est dominée par les animaux d'espaces découverts (Equus caballus, Bovinae ). Le Cerf est rare. La composition originale de cet assemblage met l'accent sur l'importance de la situation géographique (altitude, latitude) des gisements étudiés. Alors que dans le Sud-Ouest de la France les conditions climatiques se dégradaient lentement, en Basse-Auvergne vivait une faune d'espaces découverts et de caractère froid (présence de la Marmotte et du Renne).

Au-delà du seuil de la phase IX, les conditions climatiques sont dans l'ensemble froides (phases XI, XII, XIV, XVI, XVIII), entrecoupées de courtes oscillations très humides (phases XIII, XV, XVIII). Dans le Sud-Ouest et le Massif Central, les processus de cryoclastie sont prépondérants dans les abris-sous-roches alors que l'importance de la sédimentation éolienne croît dans les dépôts de recouvrement. La palynologie souligne alors le caractère steppique de ce pléniglaciaire du Würm ancien, entrecoupé de phases très humides. En Périgord, le Renne est l'espèce animale prépondérante associée au Bouquetin et à certaines formes ayant affirmé leur présence dès la phase IX (Cerf de grande taille par exemple). Dans le Massif Central, le Cheval reste omniprésent, associé au Renne, au Bouquetin, au Bison et à un Cerf de grande taille. Ces conditions pléniglaciaires vont se maintenir jusqu'à l'instauration de l'interstade würmien.

\subsection{Les séquences de Combe-Grenal et du Moustier et leur datation par thermoluminescence : pro- blèmes et questions}

\subsubsection{Combe-Grenal}

Les datations par TL de silex brûlés du gisement de Combe-Grenal (Dordogne) apportent de nouveaux éléments sur cette séquence (Bowman et De 
Tabl. 2. - Corrélations climatiques et datations TL à Combe Grenal.

Tabl. 2. - Climatic correlation and $\boldsymbol{T L}$ datings at Combe Grenal.

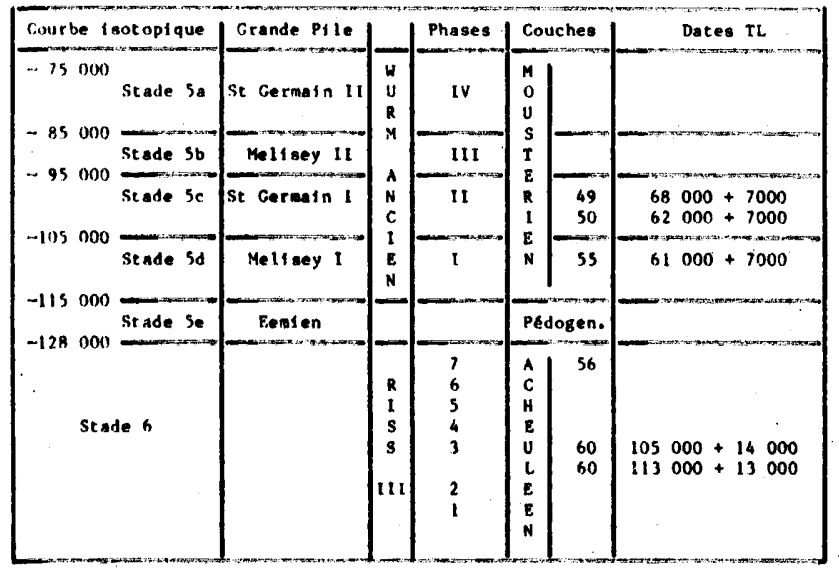

Sieveking, 1983). Non seulement ils tendent à mettre en doute la position chronologique des dépôts, mais ils conduisent également à mettre en question les corrélations précédemment établies entre toutes les séquences attribuées au Riss final et au Würm ancien au vu des données concordantes d'études pluridisciplinaires (tableau 2).

D'après Bowman et De Sieveking, ces dates placeraient la couche 60 entre 100 et 120000 B.P. selon l'intervalle de confiance à $67 \%$, soit au niveau des sous-stades $5 e$ et $5 d$ de la courbe isotopique. En considérant une erreur de deux sigmas, la même couche pourrait se situer dans le stade isotopique 6 ( - Riss III).

La partie inférieure de l'ensemble auquel correspondent les couches 55 à 49 se placerait vers 63000 B.P. (avec $4 \%$ d'erreur), soit au niveau du stade isotopique 4. Cela impliquerait que les manifestations pédologiques et l'érosion ayant affecté l'ensemble des couches 56 à 59 représentent la totalité du stade isotopique 5 et non seulement le sous-stade $5 e$; dans ce cas, les dates TL des couches 55, 50 et 49 seraient basses. $\mathrm{Si}$, par contre, ces dates sont correctes, elles suggèrent que les manifestations pédologiques et l'érosion supportées par l'ensemble $59-56$ correspondent à la fois aux sous-stades $5 \mathrm{c}$ et $5 a$.

Ce raisonnement fait abstraction des différentes données palynologiques, paléontologiques et archéologiques qui ont permis de replacer la séquence de Combe-Grenal dans le contexte régional et de proposer des corrélations avec d'autres systèmes :

- L'évolution des associations végétales et animales identifiées dans les couches 64 à 49 de Combe-Grenal est en tous points identique à celle décrite dans les séquences voisines du Pech de l'Azé
Tabl. 3. - Datations TL et U/Th du Pech de l'Azé et de La Chaise. Tabl. 3. - TL and U/Th datings at Pech de l'Azé and La Chaise.

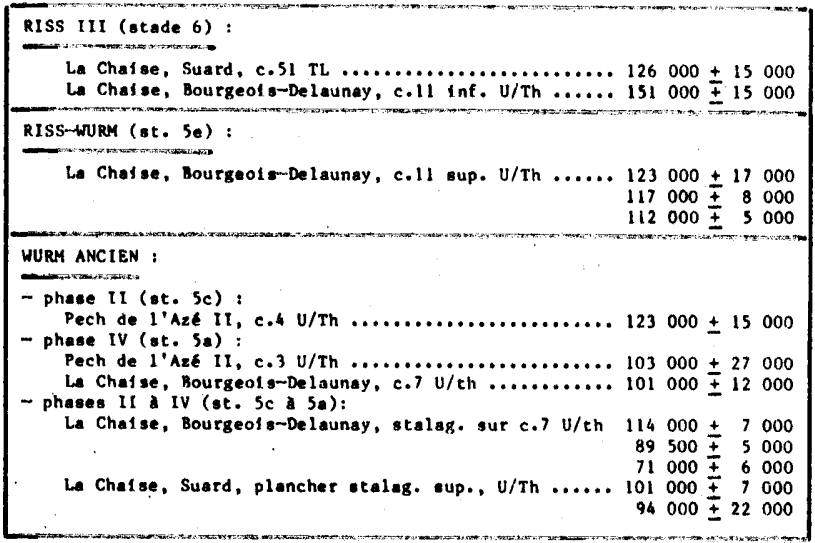

(Dordogne) et de La Chaise (Charente) pour lesquelles d'autres datations ont été obtenues (Schvoerer $e t$ al., 1977; Schwarcz et Blackwell, 1983; Blackwell, Schwarcz, Debenath, 1983). Malgré la valeur des marges statistiques qui les affectent, ces résultats doivent également être pris en considération (et sont plus conformes aux résultats attendus) (tableau 3).

- L'évolution des associations végétales de Combe-Grenal, assimilées aux sous-stades $5 \mathrm{~d}$ à $5 \mathrm{a}$, est identique à celle décelée dans les carottes marines du Golfe de Gascogne, calée par analyse isotopique, et corrélée sans équivoque avec la séquence de la Grande Pile (Turon, 1984).

- Si les manifestations pédologiques et l'érosion supportées par l'ensemble des couches 59-56 de Combe-Grenal représentent l'ensemble du stade 5, ou même les seuls termes $5 \mathrm{c}$ et $5 \mathrm{a}$, avec quels autres stades de la courbe isotopique doit-on alors corréler les phases d'importante amélioration climatique attestées dans les couches 52 à 47,43 à 42 et 41 (et leurs équivalents du Pech de l'Azé) ? Notre connaissance actuelle de la climatologie des stades isotopiques 4 et 3 ne laisse aucune place pour de telles améliorations...

- L'industrie préhistorique des couches 64 à 56 est attribuée à l'Acheuléen de faciès méridional, celle des dépôts sus-jacents au Moustérien (Bordes, 1972). Si les manifestations pédologiques supportées par les couches 59 à 56 sont rapportées aux sousstades $5 \mathrm{c}$ et $5 \mathrm{a}$, les industries acheuléennes sont d'âge nettement post-Eémien...

- A propos de dosimétrie : il n'est pas fait mention de dosimétrie pour les niveaux 49 et 55 dont les silex ont pourtant été analysés... Les silex de la couche 20 ont également servi au calcul d'un âge alors que le dosimètre avait été détruit; la dosimétrie 
ne semble pas avoir été conduite à l'emplacement initial des objets datés puisque ces derniers ont été extraits des collections bien après la fouille... (renseignements D. de Sonneville-Bordes d'après archives $\mathrm{F}$. Bordes)... L'influence du mur construit devant la coupe sur laquelle la dosimétrie a été conduite était-elle négligeable?

Nous ne mettons pas en doute les mesures physiques ayant conduit à la production des dates : sur la base des arguments exposés, nous pouvons difficilement en accepter les résultats.

\subsubsection{Le Moustier}

Nous présentons ci-après (tableau 4) la position des dépôts du Moustier dans le cadre chronoclimatique du Würm ancien d'Aquitaine et des corrélations proposées avec la courbe isotopique de l'oxygène et la zonation pollinique de la Grande Pile. En regard, sont portées les dates $\mathrm{TL}$ obtenues sur silex brûlés aimablement communiquées par $\mathrm{H}$. Valladas (1985).

Tabl. 4. - Corrélations climatiques et datations TL du Moustier. Tabl. 4. - Climatic correlations and $T L$ datings at Le Moustier.

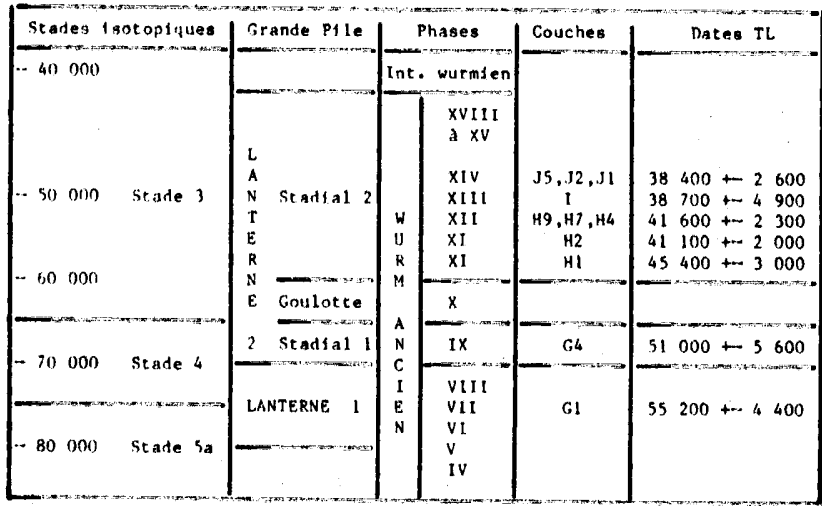

La confrontation des deux séries de données fait apparaitre deux points de désaccord :

- Les couches G4 et GI sont attribuées, au vu des données naturalistes, aux phases VII et IX du système chronologique régional. Entre autres arguments, cette attribution repose principalement sur l'étude palynologique : en effet, la couche G4 indique, pour la première fois dans le diagramme du Moustier, la présence d'herbacées héliophiles et steppiques caractéristiques (Ephedra, Galium, Poterium ) dont l'apparition est le signal du franchissement d'un seuil climatique au sein du Würm ancien. Les corrélations retenues parallélisent la phase VII avec une partie de la zone pollinique Lanterne 1 de la Grande Pile, la phase IX avec le début de la zone Lanterne 2 ("stadial I "); ces phases couvrent la fin du stade isotopique 5 a et le début du stade 4 , entre
85000 et 63000 B.P. environ. Deux hypothèses peuvent être proposées pour expliquer cette contradiction :

-. Les datations TL des couches $\mathrm{Gl}$ et $\mathrm{G} 4$ sont trop hautes de 5000 ans environ.

- Les couches G1 à G4 seraient plus récentes; dans ce cas, les manifestations d'érosion attribuées à la phase $X$ seraient en fait postérieures.

- Les couches $\mathrm{H} 1$ et $\mathrm{J}$ sont attribuées aux phases $X$ à XIV du Würm ancien par la sédimentologie, aux phases X à XII par la palynologie. Chacune de ces interprétations suppose un hiatus important entre la couche $J$ et la couche $K$ : il correspondrait aux derniers moments du Würm ancien et serait consécutif à des processus d'érosion contemporains de l'interstade würmien, le dépôt de la couche $K$ représentant les derniers moments de cet interstade.

Les dates $\mathrm{TL}$ tendent à rajeunir l'ensemble des couches $\mathrm{Hl}$ à $\mathrm{J}$ en les plaçant entre 45000 et 39000 B.P. environ, soit à la fin du Würm ancien. On est donc conduit à mettre en question les corrélations précédemment proposées et à envisager l'existence d'un hiatus de quelques millénaires entre les couches $\mathrm{G} 4$ et $\mathrm{Hl}$ (quel que soit l'âge de ces dernières). $\mathrm{Ce}$ hiatus pourrait être la conséquence des processus d'érosion que porte le sommet de G4, primitivement attribués à l'une des manifestations de la phase $X$, mais qui pourraient être bien postérieurs et contemporains par exemple de l'un des épisodes de plus forte humidité que sont les phases XIII et XV du Würm ancien; les associations végétales définies dans les couches $\mathrm{Hl}$ à $\mathrm{J}$ ne s'opposent pas à un tel " rajeunissement".

La série cohérente de dates TL obtenue au Moustier apporte donc des éléments de réflexion constructifs et stigmatise la difficulté d'apprécier l'ampleur des lacunes dans les séquences stratigraphiques.

\subsection{Corrélations}

Le coup de froid très vif de la phase I du Würm ancien a été retrouvé avec les mêmes caractéristiques dans le domaine marin voisin (Turon, 1983 et 1984). Succédant à l'interglaciaire Riss-Würm, corrélé avec le sous-stade $5 \mathrm{e}$, il doit logiquement être rapporté au sous-stade froid suivant, le sous-stade $5 \mathrm{~d}$. Il se placerait donc entre 115000 et 95000 B.P. environ. Cette détérioration brutale apparaît également avec netteté sur les diagrammes polliniques de la Grande Pile et des Echets. Elle correspond à la zone $\mathrm{C}$ des deux diagrammes et a été localement dénommée "Phase Melisey 1 ". 
Une évolution climatique identique à celle des phases II à VIII (Cf. supra) a été mise en évidence non seulement dans les sédiments du Golfe de Gascogne (Turon, 1984) mais aussi dans des séquences polliniques de la Grande Pile et des Echets. Ainsi, les phases tempérées II et IV peuvent être parallélisées respectivement avec les zones $D$ et $F$ de la Grande Pile et des Echets c'est-à-dire avec les phases Saint-Germain I et Saint-Germain II où l'on observe la même évolution des essences arbustives que dans le Sud-Ouest et le Massif-Central. La phase III plus froide qui les sépare est donc l'équivalent logique de la phase "Melisey 2 ". De la même manière, les phases $\mathrm{V}$ à VIII, qui correspondent à une progradation vers des conditions de type pléniglaciaire, se retrouvent dans les phases Ognon I-II et III ( $=$ Lanterne I).

A partir de la phase IX, l'instauration de conditions climatiques rigoureuses trouve un équivalent dans la zone $\mathbf{G}$ de la séquence des Echets et la partie supérieure de la zone Lanterne I de la Grande Pile. Les phases $X$ à XVIII se corrèlent pour leur part avec les zones $\mathrm{H}$ et $\mathrm{I}$ de la séquence des Echets et la partie inférieure de la zone Lanterne II (jusqu'à l'amélioration de Pile) de la Grande Pile : la phase $X$ (ex-« interstade Würm I-II ») trouve ainsi son équivalent avec la phase Goulotte, les phases XI à XVIII correspondent au stadial 2 de la zone Lanterne II.

Les caractéristiques climatiques des différentes phases évoquées et les datations absolues obtenues permettent de proposer les corrélations suivantes avec la courbe isotopique de l'oxygène :

- phase tempérée II = sous-stade $5 \mathrm{c}(105000$ à 95000 ans B.P. enviroń)

- phase froide $11 I=$ sous-stade $5 b(95000$ à 85000 ans B.P.)

- phase tempérée IV = sous-stade 5 a (pro parte 85000 à 75000 ans B.P. environ)

- les phases V à VIII qui traduisent une certaine instabilité climatique se situeraient à la charnière des sous-stades $5 a$ et du stade 4.

- phase froide $I X=$ stade $4(75000$ à 65000 B.P. environ)

- phases X à XVIII = partie du stade isotopique 3 (65 000-40 000 ans B.P. environ). Cependant, le caractère globalement rigoureux de cette partie du Würm ancien reste en contradiction avec la tendance plutôt tempérée de la réponse océanique durant le stade isotopique 3 ...

\section{L'INTERSTADE WURMIEN (« interstade Würm II-III 》)}

\subsection{Documents du Sud-Ouest et du Massif Central}

Les résultats récemment acquis par l'étude de dépôts de recouvrement dans le Sud-Ouest et en Basse-Auvergne précisent la signification climatique des trois termes de cet interstade qui, ailleurs, se sont traduits par des processus de pédogénèse, érosion et colluvionnement (Laville, 1969; Laville et al., 1983).

A Royat (Puy-de-Dôme, altitude $525 \mathrm{~m}$ N.G.F.), le premier terme montre une élévation progressive du boisement ( 30 à $53 \%$ ) conduisant à l'installation d'un paysage de parc dense avec pinèdes sur les sommets, chênaie claire à sous-bois de Noisetier et arbustes (Lierre, Houx, Fusain, Bourdaine), forêtgalerie (Aulne et Saule). Les dates par thermoluminescence obtenues d'une part sur les plagioclases de la coulée basaltique qui scelle les dépôts, $43000 \pm 4100,40600 \pm 3100$ (Guérin, 1983), d'autre part sur les quartz extraits des colluvions rubéfiées par la coulée $37000 \pm 3000$ (Miallier et al., 1984; Raynal et al., 1985), permettent de situer cette première étape de l'interstade würmien aux environs de 40000 B.P., peut-être un peu plus tôt, pendant l'amorce de l'événement paléomagnétique de Laschamp.

Aux Tambourets (Haute-Garonne), la première étape est également marquée par un développement du taux de boisement qui atteint $63 \%$ à son optimum, avec Chênaie mixte, Noisetier, Aulne, Frêne et arbustes (Lierre, Houx, Fusain), très nombreuses herbacées hydrophiles et fougères des lieux humides (Laville, Paquereau, Bricker, 1985).

Le deuxième terme, plus frais, se traduit aux Tambourets, par un abaissement du taux de boisement $(26 \%)$ avec disparition de la Chênaie mais persistance du Noisetier, de l'Aulne et du Saule à côté du Pin sylvestre dominant, de Graminées et de nombreuses hygrophiles.

Les associations polliniques qui, aux Tambourets, caractérisent la troisièmè étape de l'interstade rappellent celles qui sont généralement attribuées à l'amélioration des Cottés (Bastin, Lévêque et Pradel, 1976): taux de boisement atteignant $47 \%$ avec retour de tous les éléments arbustifs thermophiles et développement des hygrophiles. Elles ont également été décrites dans le gisement girondin de Camiac (Lenoir, 1984) avec un âge radiocarbone de 35100 B.P. (Ly 1 104, + $2000,-1500$ ) et sont connues en Basse-Auvergne aux environs de 34000 B.P. (33 940 $\pm 1000,33670 \pm 760$, Raynal inédit $)$. 
L'interstade würmien apparaît donc aujourd'hui comme un événement climatique majeur dont les trois termes sont repérés entre 42000 et 34000 B.P. environ.

\subsection{Corrélations}

Les corrélations avec la séquence des Echets, compte tenu de la datation radiocarbone $>34000$ B.P. donnée pour la base de la zone $\mathrm{L}$, apparaissent comme suit :

- terme 1 de l'interstade $=$ zone $J$ des Echets,

- terme 2 de l'interstade $=$ zone $\mathrm{K}$ des Echets,

- terme 3 de l'interstade $=$ zone $\mathrm{L}$ (base) des Echets.

Avec la séquence de la Grande Pile, les corrélations sont moins évidentes. Nous paralléliserions volontiers le terme 1 de l'interstade avec l'oscillation de Pile, bien que la datation radiocarbone de 49800 B.P. obtenue sur les niveaux correspondants soit un peu haute (Woillard, 1980). Cette proposition rejoint en partie celle de J.L. de Beaulieu et M. Reille qui corrèlent la zone $\mathrm{J} 2$ des Echets avec l'oscillation de Pile.

Dans cette perspective, si la phase $X$ du Würm ancien est corrélable avec l'oscillation Goulotte de la Grande Pile, et si les phases III (Arcy) et V ("Kesselt ") du Würm récent sont l'équivalent des oscillations Grand Bois 1 et 2 de la Grande Pile (datations radiocarbone compatibles), l'oscillation Charbon de cette dernière séquence pourrait représenter le terme 3 de l'interstade würmien (Les Cottés).

Les corrélations précédemment établies par comparaison avec les données du Golfe de Gascogne (Laville et al., 1983), situent l'interstade würmien à la fin du stade isotopique 3.

\section{LE STADE GLACIAIRE WURM RECENT}

En Aquitaine, les données sédimentologiques, paléontologiques et paléobotaniques s'accordent pour identifier 24 phases climatiques à l'intérieur du Würm récent, la phase $X V$ représentant l'“ interstade Würm III-IV " du système chronologique traditionnel (Delpech, 1983; Laville, 1975; Paquereau, 1976). Dans le Massif Central, les séquences stratigraphiques sont encore peu nombreuses et les corrélations n'ont été que partiellement établies avec le Sud-Ouest.

\subsection{Succession climatique}

La phase I est une étape de climat frais et humide, interrompue de deux pulsations plus douces et plus humides encore. Ces conditions se sont traduites dans les grottes et abris par la superposition de produits de gélivation et de dépôts ruisselés, par des variations du boisement (AP entre 15 et $25 \%$ ) avec Pin, Bouleau, Saule et sporadiquement Noisetier en proportions variables. Les hygrophiles, toujours abondantes, indiquent une humidité constante. Dans le Sud-Ouest, le Renne domine la faune et est accompagné de quelques formes de climat humide.

La phase II, très froide et plus sèche est exclusivement représentée par une sédimentation de type périglaciaire. Un abaissement du boisement (AP $<10 \%$ ) permet l'installation de zones ouvertes très sèches, peuplées d'héliophiles. Dans le Sud-Ouest, le Renne, largement dominant, n'est accompagné d'aucune forme forestière. En Velay par contre, le Cheval constitue l'essentiel des associations fauniques (y compris pendant la phase III) accompagné de grands bovidés.

On assiste ensuite, jusqu'à la phase VIII incluse, à l'alternance d'épisodes d'adoucissement et d'épisodes plus rigoureux.

Les phases III, $\mathrm{V}$ et VII sont marquées par la raréfaction des produits de gélivation et la mise en place de dépôts ruisselés (phases III et $V$ ) ou colluviés (phase VII). Il leur correspond une élévation sensible du boisement $(A P=25$ à $40 \%)$ mais qui n'atteint jamais celui de l'interstade würmien : parmi les feuillus, le Noisetier est dominant avec des éléments des zones fraîches et humides (Tilleul, Frêne, Aulne, Saule), caractérisant des boisements clairs et des forêts galeries. Une forte humidité est indiquée par le développement des herbacées hygrophiles. Dans ce contexte, l'intrusion d'une faible pulsation plus froide au cours de la phase VII, attestée à la fois par la sédimentologie (produits de gélivation plus nombreux) et la palynologie (AP < $20 \%$ ) constitue l'un des marqueurs chronologiques du Würm récent en Aquitaine. Le Renne, toujours présent, est associé en proportions variables à des formes forestières.

Les données de la sédimentologie et de la palynologie identifient les phases IV, VI et VIII à des étapes de climat particulièrement rigoureux : sédimentation quasi exclusive de produits de gélivation, taux de boisement oscillant entre 5 et $10 \%$ avec Pin sylvestre seul, fort pourcentage des Composées cichoriées et des Xérophiles, en particulier Ephedra et Galium caractérisant des paysages de steppes. Le Renne reste dominant, mais est accompagné de quelques formes de forêt lors de la phase IV, et lors 
de la phase VI, du Bœuf primitif, d'Equus Hydruntinus, du Chevreuil, et du Sanglier dans les gisements de Dordogne. En Basse-Auvergne, l'instauration des conditions steppiques est attestée dès 28 000-27 000 B.P. (Miallier et al., 1984, Raynal et al., 1985).

Les différentes données concourrent à situer le franchissement d'un seuil climatique avec la phase VIII dans le Sud-Ouest. Entre autres arguments, on notera que les feuillus thermophiles que l'on retrouve à chacune des améliorations climatiques jusqu'à la phase VII incluse ne réapparaitront qu'avec la phase XXI. Parallèlement, à partir de la huitième phase climatique, les populations de Renne d'Aquitaine apparaissent de moins haute stature que précédemment, particularité mise en relation avec un environnement moins favorable qui se maintiendra jusqu'à la phase XX incluse (Delpech, 1983).

Le caractère globalement froid de toute la période correspondant aux phases IX à XIV est indiqué par la prépondérance des produits de gélivation à tous les niveaux de la séquence correspondante. Seuls trois dépôts de granulométrie plus fine marquent trois épisodes de faible amélioration thermique et de plus forte humidité (phases IX, XI et XIII). Dans les niveaux correspondant à ces trois phases, le taux de boisement ne dépasse pas $22 \%$ avec Pin sylvestre dominant, quelques noisetiers et bouleaux accompagnés de nombreuses hygrophiles.

Les phases X, XII et XIV présentent sur le plan palynologique, des caractéristiques identiques à celles des phases VI et VIIl précédentes. En Aquitaine, le Renne constitue toujours l'essentiel de la faune et présente, comme lors de la phase VIII, une stature relativement petite. A ses côtés, on note le Mammouth, le Cheval, le Bouquetin, le Chamois, de grands Bovidés et le cerf.

Ainsi que nous l'avons indiqué précédemment, les conditions acquises lors de la phase VIII avec laquelle un seuil climatique est franchi, se maintiendront ensuite jusqu'à la phase $\mathrm{XX}$ que des données radiométriques cohérentes permettent de paralléliser avec la fin du Dryas 1 .

Dans ce contexte pléniglaciaire, les phases XV $(=$ "interstade Würm -III-IV" = Laugerie), XVII (Lascaux) et XIX (Pré-Bölling) n'apparaissent que comme l'expression de crises d'humidité excessive, thermiquement peu marquées. En Aquitaine, l'augmentation du boisement contemporain (phase XV : $\mathrm{AP}=30 \%$, phase XVII : AP $=20 \%$, phase XIX : AP $<20 \%$ ) ne correspond qu'au développement du Noisetier, de l'Aulne et du Saule aux côtés du Pin sylvestre dans un paysage largement dominé par les herbacées hygrophiles. Le Renne constitue aux mêmes niveaux, l'essentiel de la faune et conserve toujours une stature relativement réduite. Dans le
Massif Central, les données de la zone montagneuse du Velay révèlent également le caractère froid et humide de "l'interstade Würm III-IV" (= Laugerie), la faune restant dominée par le Bouquetin (Debard et al., 1976).

Les phases XVI, XVIII et XX sont systématiquement marquées par l'accroissement des herbacées héliophiles et steppiques et le taux de boisement ne dépasse pas $10 \%$. La sécheresse du climat est surtout confirmée par l'apparition de l'Antilope Saïga lors de la phase XVI et son développement lors des phases XVIII et XX; cet "épisode à Saiga " constitue l'un des marqueurs chronologiques de cette période en Aquitaine (Delpech, Laville, Rigaud, sous presse); il a été retrouvé en BasseAuvergne (travaux F. Delpech in Raynal, 1984).

Les données du domaine continental suggèrent qu'avec la phase XX (Dryas l) se termine le pléniglaciaire. A partir de ce moment là, s'amorce la dynamique climatique qui sera celle de l'Holocène : la phase XXI (équivalent du Bölling) et surtout la phase XXIII (Alleröd) semblent en effet bien marquées dans nos régions et le Dryas II qui les sépare, s'il est perceptible apparaît court et de faible intensité. C'est en effet avec le Bölling que réapparaissent les grands feuillus thermophiles, qui, après une brève disparition lors du Dryas II, conduiront au développement de la Chênaie mixte lors de l'Alleröd. C'est au même moment (Bölling) que correspond le développement des troupeaux de grands Ongulés (Bison, Cheval). Le Renne est encore présent et acquiert à nouveau dès ce moment là, la stature qui était la sienne jusqu'à la phase VII. Il disparaîtra de nos régions au cours de l'Alleröd, alors qu'apparaîtront, plus nombreuses, les formes de climat tempéré et humide, telles que Cerf, Chevreuil et Sanglier.

Dans cette évolution climatique qui tend progressivement à des conditions interglaciaires, le Dryas III ( = phase XXIV du système régional) prend le caractère intrusif et brutal d'une crise climatique (Rognon, 1983).

\subsection{Corrélations}

Compte tenu des caractéristiques des différents épisodes climatiques que nous venons de décrire, les trois épisodes d'élévation thermique et de plus forte humidité que représentent les phases III, V et VII du Würm récent peuvent être respectivement corrélés avec les "interstades " d'Arcy, de "Kesselt " et de Tursac de la zonation pollinique établie par A. Leroi-Gourhan (1977).

Il convient cependant de noter que la prise en compte de 76 datations ${ }^{14} \mathrm{C}$ réalisées en Aquitaine 
sur des dépôts correspondant aux phases I à XIV ne permet pas de situer ces épisodes dans le temps à moins de 1000 ans près. Cette constatation nous a conduits à mettre en doute la validité des limites chronologiques des épisodes d'Arcy, "Kesselt " et Tursac fixés par A. Leroi-Gourhan (Laville, Delpech, Rigaud, 1985). L'absence de manifestations attribuables aux phases IX, XI et XIII dans cette zonation révèle vraisemblablement son caractère incomplet à ce niveau de la séquence, puisque la zonation paléoclimatique de l'équivalent chronologique de la période inter Tursac-Laugerie en Belgique montre également la succession de légères améliorations climatiques (Haesaerts, 1984).

Nous avons précédemment établi que le stade Würm récent correspondait sensiblement au stade isotopique 2 (Laville et al., 1983). Il se corrèle avec les zones $\mathrm{L}$ (sommet), $\mathrm{M}, \mathrm{N}, \mathrm{O}$ et $\mathrm{P}$ de la séquence des Echets. Dans la séquence de la Grande Pile, il commence avec le stadial 4 de la zone Lanterne 2 et se poursuit avec les épisodes Grand-Bois I et II, équivalents possibles d'Arcy et "Kesselt " et de nos phases III et V; les phases ultérieures se parallèlisent avec les différents termes de la zone Lanterne III et les équivalences entre les épisodes d'amélioration seraient les suivantes :

- phase VII; $=$ Tursac $=$ Marcoudan 1,

- phase IX Laugerie = Marcoudan 2,

-. phase $\mathrm{XI}=$ Lascaux $=$ Marcoudan 3.

\section{CONCLUSIONS}

Plusieurs conclusions découlent de l'exposé des manifestations climatiques reconnues sur la base du décryptage des modifications des paléoenvironnements physiques et biotiques et de leur position chronologique étayée par des méthodes de datation objective.

\subsection{Enchaînement des phénomènes}

A la suite du pléniglaciaire du Riss récent, l'interglaciaire Riss-Würm (130 000-115000 B.P. environ) introduit une période majeure de biostasie, caractérisée par une relative stabilité morphologique et l'instauration d'un système climatique à grande inertie.

Entre 115000 et 105000 B.P. environ, une crise climatique de forte intensité rompt l'équilibre climatique. Cette rupture, particulièrement bien enregistrée par les milieux physiques et biotiques, est le signal de l'établissement du système climatique würmien. Elle marque le début du Würm ancien.

L'inertie du système interglaciaire précédent est cependant telle que l'on note ensuite, à plusieurs reprises, un retour momentané de conditions tempérées, d'amplitude décroissante. Ainsi, entre 105000 et 75000 B.P. environ, des oscillations progressives vers le maximum glaciaire provoquent une grande instabilité des régimes morphosédimentaires (tendance rhexistasique) et enclenchent un processus de modification des associations animales et végétales. Vers 70000 B.P., un seuil climatique est franchi qui consacre l'instauration des conditions pléniglaciaires. Ces conditions, entrecoupées de crises d'humidité, se maintiendront jusqu'à "l'interstade würmien " vers 40000 B.P. environ, qui marque la fin du Würm ancien.

Au même titre que l'interglaciaire précédemment, mais avec une moindre intensité et durée, l'interstade würmien rompt l'équilibre climatique pléniglaciaire du Würm ancien. Le terme 2 de l'interstade pourrait représenter, toute proportion gardée, le signal du début du stade suivant : dans ce cas, le terme 3 (Les Cottés), représenterait déjà la seconde phase (tempérée) du Würm récent. Cette hypothèse demande confirmation.

Entre 35000 et 15000 B.P., la succession des événements climatiques du Würm récent correspond à un scénario identique à celui décrit pour le Würm ancien, mais de durée plus contractée. Après une période d'instabilité plus courte, les conditions pléniglaciaires se réalisent après le franchissement d'un seuil, plus ou moins tardif selon les paramètres géographiques, et se maintiendront jusqu'au déclenchement de la dynamique de déglaciation.

\subsection{Nature des transitions}

Dans l'état actuel de nos connaissances, les transitions d'un stade glaciaire à la période d'amélioration suivante sont généralement brutales : il en est ainsi pour le passage du Riss III au Riss-Würm, pour celui du Würm ancien à l'interstade würmien. Par contre, du Würm récent à l'Holocène, la transition apparaît plus progressive.

L'instabilité des conditions climatiques du début du Würm ancien et du Würm récent montre par contre qu'il est plus difficile de rompre un équilibre climatique tempéré (interglaciaire ou interstadiaire). Cette observation est d'ailleurs transposable aux périodes antérieures, pour ce que nous en connaissons (Texier et al., 1983), mais possédons-nous des outils de résolution suffisante pour analyser de tels processus? 


\subsection{Portée des faits reconnus}

La succession des faits reconnus, retrouvée dans le proche domaine océanique, dépasse donc le cadre régional. Elle correspond manifestement à une mécanique climatique généralisée de l'hémisphère nord. D'ailleurs, les séquences continentales plus orientales (Grande Pile, Les Echets) établissent une succession identique. Fondée sur une approche pluridisciplinaire, elle établit avec force que les modifications subies par les milieux physiques et biotiques enregistrées par les sédiments continentaux, avec une fidélité plus ou moins bonne selon leur nature, ne sont généralement pas aléatoires et transcrivent bien une réalité paléoclimatique. Elle démontre qu'en l'absence de grande séquence en milieu privilégié (lac, tourbière...), il est possible d'établir une histoire paléoclimatique régionale pourvu que l'on dispose d'un grand nombre de stratigraphies corrélables et de datations objectives permettant de relativiser l'ampleur des manifestations reconnues. Enfin, elle apporte selon nous des éléments déterminants au débat introduit depuis quelques années en matière de chronologie et de terminologie du Pléistocène supérieur : le dernier stade glaciaire commence bien vers 115000 B.P. et dure une centaine de millénaires.

\section{BIBLIOGRAPHIE}

Avertissement: les références qui ne figurent pas dans cette bibliographie sont citées dans l'ouvrage Paléoclimats, Actes Coll. A.G.S.O. Bordeaux, mai 1983, Bull. Inst. Géol. Bassin d'Aquitaine. Bordeaux $\mathrm{n}^{\circ} 34$ et C.N.R.S. Cahiers du Quaternaire, $\mathrm{n}^{\circ}$ spécial, 1983, 249 p.

Beaulieu J.-L. de, Reille M., 1984. - The pollen sequence of Les Echets (France) : a new element for the chronology of the Upper Pleistocene. Géographie physique et quaternaire, vol. XXXVIII, $\mathrm{n}^{\circ}$ 1, p. 3-9.

Blackwell B., Schwarcz H., Debenath A., 1983. Absolute Dating of Hominids and Palaeolithic Artefacts of the cave of La Chaise-de-Vouthon (Charente), France. Journal of Archaeological Science, 10, p. 493-513.

Bowman S.G.E., De Sieveking G., 1983. -- Thermoluminescence dating of burnt flint from CombeGrenal. In : Third specialist Seminar on TL and ESR dating, July 1982. PACT, 9, p. 253-268.

Debard E. et al., 1976. - Conclusions générales sur l'environnement de la Grotte de Cottier (Retour- nac, Haute-Loire) et sur la position chronologique de son remplissage. Nouv. Arch. Mus. Hist. nat. Lyon, fasc. 14, p. 49-52.

Delpech F., 1983. - Les faunes du Paléolithique supérieur dans le Sud-Ouest de la France. Cahiers du Quaternaire, C.N.R.S. éd., 6, 473 p.

Delpech F., 1984. - Evolution des associations d'ongulés durant le troisième stade würmien : implications paléoclimatiques. $10^{e}$ R.A.S.T., Bordeaux, p. 177.

Delpech F., 1984. - Les Ongulés en Périgord et Nord-Ouest du Quercy durant le Würm III, Chronoclimatologie, paléobiogéographie, palethnologie. Geobios, $\mathrm{n}^{\circ}$ 17, fasc. 5, p. 531-548, 1 fig., 14 tabl.

Delpech F., Laville H., Rigaud J.-P., 1983. Chronologie et environnement climatique du Paléolithique supérieur dans le Sud-Ouest de la France. Actes des travaux de la Commission 10 de I'U.I.S.P.P., réunion de Leon (Espagne), mars 1983, sous presse.

Fedoroff N., Tursina, 1984. - Micromorphologie des sols lessivés de France et des sols dernopodzoliques d'Union Soviétique. I.N.R.A., sous presse.

Guérin G., 1983. - Thermoluminescence des plagioclases. Méthode de datation du volcanisme. Applications au domaine volcanique français: Chaîne des Puys, Mont-Dore et Cézalier, Bas Vivarais. Thèse d'Etat, Université P. et M. Curie, Paris.

Haesaerts P., 1984. - Aspects de l'évolution du paysage et de l'environnement en Belgique au Quaternaire. In : Peuples chasseurs de la Belgique préhistorique dans leur cadre naturel. Publ. de l'Institut royal des Sciences naturelles de Belgique, p. 27-39.

Laville H., Delpech F., Rigaud J.-Ph., 1985. - Sur la zonation pollinique du Pléistocène récent : les précisions du domaine aquitain. Actes Coll. Palynologie archéologique, CNRS Ed, Notes et Monographies techniques du C.R.A. $n^{\circ} 17$, p. 245-257.

Laville H., Raynal J.-P., Texier J.-P., 1984. Interglaciaire... ou déjà glaciaire? Bull. Soc. préhist. fr., 81, 1984, $\mathrm{n}^{\circ} 1$, p. 8-11.

Laville H., Paquereau M.-M., Bricker H., 1985. Précisions sur l'évolution climatique de l'interstade würmien et du début du Würm récent : les dépôts du gisement castelperronien des Tambourets (Haute-Garonne) et leur contenu pollinique. C.R. Acad. Sc. Paris, t. 301, Série II, n 15, 1985, p. 1 137-1 140.

Lenoir M., 1983. - Le Paléolithique des basses vallées de la Dordogne et de la Garonne. Thèse de 
Doctorat d'Etat ès-Sciences, Univ. Bordeaux I, 702 p., 44 tabl., 445 fig., 17 cartes.

Miallier D., 1982. -- L'usage des détecteurs solides de traces dans le cadre de la datation par thermoluminescence. Thèse de $3^{\mathrm{e}}$ Cycle, Université de Clermont II, $107 \mathrm{p}$.

Miallier D., Fain J., Sanzelle S., Raynal J.-P., Daugas J.-P., Paquereau M.-M. (1984). - Datation du volcanisme quaternaire du Massif Central français par la méthode des inclusions de quartz en thermoluminescence et comparaison avec d'autres approches. $10^{e}$ R.A.S.T., Bordeaux, 2-6 avril 1984, p. 396.

Prat F., Suire C., 1971. - Remarques sur les Cerfs contemporains des deux premiers stades würmiens. Bull. Soc. préhist. fr., C.R.S.M., no 3, p. 75-79.

Raynal J.P., 1984. - Chronologie des basses terrasses de l'Allier en Grande Limagne (Puy-de-Dôme, France), Bull. Ass. Fr. Et. Quat., $n^{\text {os }} 17,18,19$, p. 79-84.

Raynal J.-P., Daugas J.-P., Paquereau M.-M., Amprimoz F.-X., 1981. - Arguments en faveur d'un âge rissien pour le fossile humain découvert en 1876 au lieu-dit Les Rivaux, à Espaly (HauteLoire). Cr. Acad. Sc. Paris, t. 292, série II, p. I 501-1 504, 1 tabl., I fig.

Raynal J.-P., Paquereau M.-M., Daugas J.-P., Miallier D., Fain J., Sanzelle S., 1985. Contribution à la datation du volcanisme du Massif Central français par la thermoluminescence des inclusions de quartz et comparaison avec d'autres approches: implications chronostratigraphiques et paléoenvironnementales. Bull. Ass. Fr. Et. Quat. 4, p. 183-207.
Raynal J.-P., Daugas J.-P., Paquereau M.-M., Guadelli J.-L., Marchianti D., Miallier D., Fain J., Sanzelle S., 1984. - Le maar de Saint-Hippolyte (Puy-de-Dôme, France) : datation par thermoluminescence, flores et faunes fossiles, présence humaine, climatochronologie et dynamique du système paléo-lacustre. Actes du Jubilé H. Pelletier, Revue des Sciences Naturelles d'Auvergne, Nouvelle série, vol. 50, fasc. 1-2-3-4, p. 97-114.

Schvoerer M., Rouanet J.-F., Navailles H., Debenath A., 1977. - Datation par thermoluminescence de restes humains antéwürmiens de l'abri Suard, à la Chaise de Vouthon (Charente). C.R. Acad. Sc. Paris, 284, p. 1 979-1 982.

Schwarcz H., Blackwell B., 1983. - Absolute dating of Hominids and Paleolithic Artifacts of the cave of La Chaise-de-Vouthon (Charente), France. Journal of Archaeological Science, 10, p. 493-513.

Turon J.L., 1984. - Direct land/sea correlations in the last interglacial complex. Nature, vol. 309, $n^{\circ} 5970$, p. 673-676.

Valladas H., 1985. - Datation par thermoluminescence de gisements moustériens du Sud de la France. Thèse de Doctorat ès-Sciences, Université de Paris VI.

Woillard G., 1978. - Grande Pile Peat Bog: a continuous Pollen Record for the last 140000 years. Quaternary Research, 9, p. 1-21.

Woillard G., 1980. - Palynology and Radiocarbon Datings at Grande Pile : a remised Radiocarbon time scale for the late Pleistocene. In : Quaternary Glaciations in the Northern Hemisphere, Report $n^{\circ} 7$, p. 245-252. 